\title{
Reversal of reserpine-induced depression and cognitive disorder in zebrafish by sertraline and Traditional Chinese Medicine (TCM)
}

Shuhui Zhang ${ }^{1 \dagger}$, Xiaodong Liu ${ }^{1 \dagger}$, Mingzhu Sun ${ }^{3 \dagger}$, Qiuping Zhang ${ }^{2 \dagger}$, Teng Li ${ }^{3}$, Xiang Li ${ }^{1}, J_{i a ~ X u^{2}}$, Xin Zhao ${ }^{3^{*}}$, Dongyan Chen ${ }^{2^{*}}$ and Xizeng Feng ${ }^{1^{*}}$

\begin{abstract}
Background: With increased social pressure, individuals face a high risk of depression. Subsequently, depression affects cognitive behaviour and negatively impacts daily life. Fortunately, the Traditional Chinese Medicine Jia Wei Xiao Yao (JWXY) capsule is effective in reducing depression and improving cognitive behaviour.

Methods: The constituents of JWXY capsule were identified by ultra-performance liquid chromatography and quadrupole time-of-flight mass spectrometry analyses. We analysed behaviours of depression-like zebrafish in the novel tank with an automatic 3D video-tracking system and conducted the colour preference test, as well detected physiological changes after sertraline and JWXY capsule treatments.

Results: Both sertraline and JWXY capsule rescued the decreased locomotive behaviour and depression phenotype of zebrafish caused by reserpine. JWXY capsule especially improved the inhibited exploratory behaviour caused by reserpine. In addition, with the onset of depressive behaviour, zebrafish exhibited alterations in cognitive behaviour as indicated by colour preference changes. However, compared with sertraline, JWXY capsule was more efficaciously in rescuing this change in the colour preference pattern. Moreover, an increased level of cortisol, increased expression of tyrosine hydroxylase $(\mathrm{TH})$ and decreased monoamine neurotransmitters, including serotonin (5-HT) and noradrenaline, were involved in the depressive behaviours. In addition, sertraline and JWXY capsule rescued the depressive phenotype and cognitive behaviour of zebrafish by altering the levels of endogenous cortisol and monoamine neurotransmitters.
\end{abstract}

Conclusions: JWXY capsule was more effectively than sertraline in rescuing reserpine-induced depression and cognitive disorder in zebrafish. Potentially, our study can provide new insights into the clinical treatment of depression and the mechanism of action of JWXY capsule.

Keywords: Depression behaviour, TCM, Colour preference, Monoamines, Zebrafish

\footnotetext{
*Correspondence: zhaoxin@nankai.edu.cn; chendy@nankai.edu.cn; xzfeng@nankai.edu.cn

†Shuhui Zhang, Xiaodong Liu, Mingzhu Sun and Qiuping Zhang

contributed equally to this work

${ }^{1}$ State Key Laboratory of Medicinal Chemical Biology, The Key Laboratory

of Bioactive Materials, Ministry of Education, College of Life Science,

Nankai University, Tianjin 300071, China

${ }^{2}$ Tianjin Key Laboratory of Tumor Microenvironment and Neurovascular

Regulation, Department of Histology and Embryology, School

of Medicine, Nankai University, Tianjin 300071, China

${ }^{3}$ The Institute of Robotics and Automatic Information Systems, Nankai

University, Tianjin 300071, China
}

(c) The Author(s) 2018. This article is distributed under the terms of the Creative Commons Attribution 4.0 International License (http://creativecommons.org/licenses/by/4.0/), which permits unrestricted use, distribution, and reproduction in any medium, provided you give appropriate credit to the original author(s) and the source, provide a link to the Creative Commons license, and indicate if changes were made. The Creative Commons Public Domain Dedication waiver (http://creativecommons.org/ publicdomain/zero/1.0/) applies to the data made available in this article, unless otherwise stated. 


\section{Background}

Major depressive disorder (MDD), one of the most common brain disorders, usually has a high rate of comorbidity with other psychiatric disorders [1]. Depressive disorder, characterised by decreased activity, a significant and lasting low mood, and slowed thinking and cognitive function [2,3], markedly reduces quality of life. Psychiatric disorders such as psychosis, depression, and other mood disorders may have multigenic and multifactorial aetiologies [4]. Fortunately, improvements in the diagnosis and treatment of depression are increasing. Monoamines play a key role in the regulation of brain functions in animals and humans [5]. Monoamine neurotransmitters, including serotonin (5-HT), dopamine (DA) and noradrenaline (NA), are implicated in the regulation of a large number of processes, such as motor control, social behaviour, cognition, sleep, appetite, and anxiety in vertebrates [6-9]. In zebrafish, 5-HT and DA are the two most studied monoamines [10, 11]. Serotonin (5-hydroxytryptamine, 5-HT) serves as both a neurotransmitter and hormone; in higher vertebrates, 5-HT acts throughout the body, including the central nervous system (CNS), peripheral nervous system, cardiovascular system, and endocrine system; it also participates in sensory perception and many behaviours [12]. Serotonin is involved in many behavioural functions, including the organization of defence, and its putative pathological correlate, anxiety and stress disorders [13]. Anxietylike behaviour positively correlates with 5 -HT content in the novel tank test [14]. Stress levels can be measured by the whole-body cortisol concentration [15]. Some compounds cause Parkinson's disease-like behaviour due to decreased dopamine levels and locomotor activity [16, 17]. Thus, the study of monoamine neurotransmitters in the brain is indispensable for the treatment of depression.

For depression, the most widely used therapy is antidepressants, including monoamine oxidase inhibitors (MAOIs), tricyclic antidepressants (TCAs), serotonin and norepinephrine reuptake inhibitors (SNRIs) and selective serotonin reuptake inhibitors (SSRIs) [18]. For example, sertraline is one a SSRI. Although these antidepressant drugs are effectively relieve depression, they have several concerning side effects, such as headache, agitation or sedation, vomiting, and fatigue $[19,20]$. Therefore, identifying a better antidepressant is necessary; this need has led researchers to focus on natural medicine, including Traditional Chinese Medicine (TCM). TCM has a long history of prevention and treatment of depression dating as far back as 2000 years ago. When treating depression, TCM starts at the whole-body level, considering not only the psychological problems that result from a patient's nervous system disorder but also the changes in the Zang-Fu organs, qi and blood [18]. TCM, such as Jia Wei
Xiao Yao (JWXY) capsule, can provide a reliable clinical curative effect comparable to that of Western medicine. In addition, TCM is much more affordable and has fewer side effects. However, a lack of rigorous clinical research has counteracted the unique advantage of TCM and seriously impeded its worldwide popularization and application. JWXY capsule can soothe the liver and reduce heat, strengthen the spleen and nourish the blood. Based on experiences with TCM, JWXY capsule exerts various actions, including soothing the liver and improving the circulation of qi to relieve depression. In China, JWXY capsule has been commonly recognized as a safe and effective prescription in the treatment of depressive disorder [18, 21-23]. However, the effects and mechanism of action of JWXY capsule remain poorly understood.

In the literature, several assays have been reported to measure behavioural learning changes in adult zebrafish such as the rotating escape test, bite test, novel tank test, place preference test, T-maze, plus maze and Y-maze assays [24, 25]. Most of tools used to assess learning and memory in animal models involve visual stimuli, including colour preferences. Zebrafish can discriminate colours and display spontaneous approach or avoidance behaviours. Some studies support colour-based learning and memory paradigms or experiments involving aversion, anxiety or fear in zebrafish [26, 27]. Zebrafish show a preference for blue and green and avoided yellow and red [28]. The zebrafish visual system includes retinas with cones sensitive to red, green, blue, and ultraviolet; moreover zebrafish are diurnal animals, which makes them an ideal model for developing research on cognitive responses to visual signals $[29,30]$.

In cognitive research, the zebrafish has become increasingly popular and has advantages in behavioural brain research due to its elaborate brain structure, simplicity and neurochemistry, which offers translational relevance to humans [31-33]. In addition, the zebrafish is an ideal and promising model organism for pharmacology $[4,18$, 34-36], disease [35, 37], embryology and development studies [38,39] because it shares many genes, protein products and molecular pathways with mammals [40]. There are also studies on the relationship between emotion regulation and colour preference in zebrafish [41]. Zebrafish may become a translationally relevant study species for the analysis of the mechanisms of learning and memory changes associated with psychopharmacological treatment of anxiety/depression [42].

Compared with 2D approaches, a 3D approach improves data integrity by using two videos and may help reduce the number of experimental subjects. We used two cameras covering the dorsal and lateral view to record fish behaviour in a novel tank. A 3D approach integrates the position information from the top and 
front views, which is essential to measure depressionlike behaviour in zebrafish [43]. 2D approaches have also played a pivotal role in elucidating the neurobehavioural underpinnings of fish behaviour [43]. Hence, we utilized a camera from the top view to record the preference of zebrafish for different colours after pharmacological manipulations.

Reserpine causes depression by depleting monoamines and is widely used to induce depression-like phenotypes by pharmacological manipulation in zebrafish [5]. Therefore, in this study, we performed comparative analysis of the curative effect of sertraline and JWXY capsule treatment for reserpine-induced depression-like behaviour in zebrafish by examining behaviour and the concentrations of three monoamine neurotransmitters and the hormone cortisol. Sertraline and JWXY capsule rescued depressive behaviour and colour preference, accompanied by changes in monoamines and cortisol. The purpose of our study was to evaluate the effects of sertraline and JWXY capsule on behaviour, cognitive ability and biochemical parameters in zebrafish with depression induced by reserpine.

\section{Methods}

\section{Zebrafish}

Zebrafish ( $\mathrm{AB}$ strain) were maintained in a fish-farming system at the State Key Laboratory of Medicinal Chemical Biology, Nankai University. The room was maintained at a constant temperature of $28.5^{\circ} \mathrm{C}$ on a constant light cycle ( $14 \mathrm{~h}$ light $/ 10 \mathrm{~h}$ dark), and the water $(\mathrm{KCl} 0.05 \mathrm{~g} / \mathrm{L}$, $\mathrm{NaHCO}_{3} 0.025 \mathrm{~g} / \mathrm{L}, \mathrm{NaCl} 3.5 \mathrm{~g} / \mathrm{L}$, and $\mathrm{CaCl}_{2} 0.1 \mathrm{~g} / \mathrm{L}$ ) was circulated continuously. The zebrafish were fed freshly hatched brine shrimp twice daily. All of the experimental protocols and procedures involving zebrafish were approved by the Committee for Animal Experimentation of the College of Life Science at Nankai University (no. 2008) and were performed in accordance with the NIH Guide for the Care and Use of Laboratory Animals (no. 8023, revised in 1996).

\section{Behavioural test apparatuses and behavioural parameters}

Behavioural apparatuses were designed according to previous studies [44, 45]. A novel tank, composed of transparent Plexiglass, was a $5 \mathrm{~L}$ rectangular box $(23 \mathrm{~cm}$ length * $15 \mathrm{~cm}$ width * $15 \mathrm{~cm}$ depth) used to assess the depressive behaviour of zebrafish. We divided the tank into two equal horizontal portions virtually by marking a midline on the outside walls. The region above this midline indicated the "top" of the novel tank, while the area below indicated the "bottom" of the novel tank. The novel tank was placed over a light source, a light-emitting diode (LED) array, with an acrylic diffuser located above the tank. The light source was composed of white light (500 lux) arrays and a transparent platform. Two charge-coupled device (CCD) cameras (MV-VS078FM, Microvision, 10 frames/s) were placed to obtain the top (dorsal) view and side (lateral) view of the moving zebrafish (Fig. 1b). The offset cross maze and T-maze (Fig. 1c) were designed based on previous research and composed of transparent Plexiglass. Every arm of the offset cross maze is $20 \mathrm{~cm}$ * $8.8 \mathrm{~cm}$. The sides of the four arms are covered with four different colours (blue, green, red and yellow) made from polypropylene. The centre Section $(8.8 \mathrm{~cm} * 8.8 \mathrm{~cm})$ of the cross maze is a starting place for the fish indicated by None. The two opposite arms of the maze are $20 \mathrm{~cm} * 8.8 \mathrm{~cm}$ each and are covered with two different colours (blue and yellow) of polypropylene on the sides. The last arm is $20 \mathrm{~cm} * 8.8 \mathrm{~cm}$ and uncoloured. The last arm and centre Section $\left(8.8 \mathrm{~cm}^{*} 8.8 \mathrm{~cm}\right)$ of the T-maze is a starting place for the fish indicated by No. The maze is $10 \mathrm{~cm}$ deep and filled with $6.5 \mathrm{~cm}$ system water. A CCD camera (MV-VS078FM, Microvision, 10 frames/s) was fixed above the maze to obtain the top view of the moving zebrafish (Fig. 1c). A daylight lamp (500 lux) or natural light served as the light source. All apparatuses rested on a level, stable surface and were placed in a relatively sound-proof room to minimize the effect of noise when behavioural tests were conducted. A big black cloak forming a space covered all the experimental apparatuses to eliminate environmental interference.

Briefly, the behavioural parameters were defined according to the literature and previous research $[44,45]$. The definitions of behavioural parameters that described depression in the novel tank are shown in Table 1. The definitions of behavioural parameters that described colour preferences in the maze are provided in Table 2.

\section{Chemical and experimental design}

Reserpine (purity $\geq 98.0 \%$ ) was purchased from Shanghai Macklin Biomedical Co., Ltd. The reserpine concentration of $40 \mu \mathrm{g} / \mathrm{mL}$ in this study was chosen based on previous research concerning the effective doses of reserpine for the depressive behaviour of zebrafish $[5,45]$. Sertraline hydrochloride (purity $>98.0 \%$ ) was purchased from TCI Co., Ltd. (Shanghai, China). Preliminary experiments proved that the effective concentration of sertraline hydrochloride was $0.1 \mu \mathrm{g} / \mathrm{mL}$. The experimental doses of reserpine $(40 \mu \mathrm{g} / \mathrm{mL})$ or sertraline hydrochloride $(0.1 \mu \mathrm{g} / \mathrm{mL})$ were obtained by weighing and adding dry powder to system water. JWXY capsule (Z10960066) was purchased from Sichuan Baoxing Pharmaceutical Co., LTD (Sichuang, China). The composition of JWXY capsule is as follows: Bupleuri Radix, Angelicae Sinensis Radix, Paeoniae Radix Alba, Atractylodis Macrocephalae Rhizoma (stir-baking with bran), Poria, Glycyrrhizae Radix Et Rhizoma, Menthae Haplocalycis Herba, 


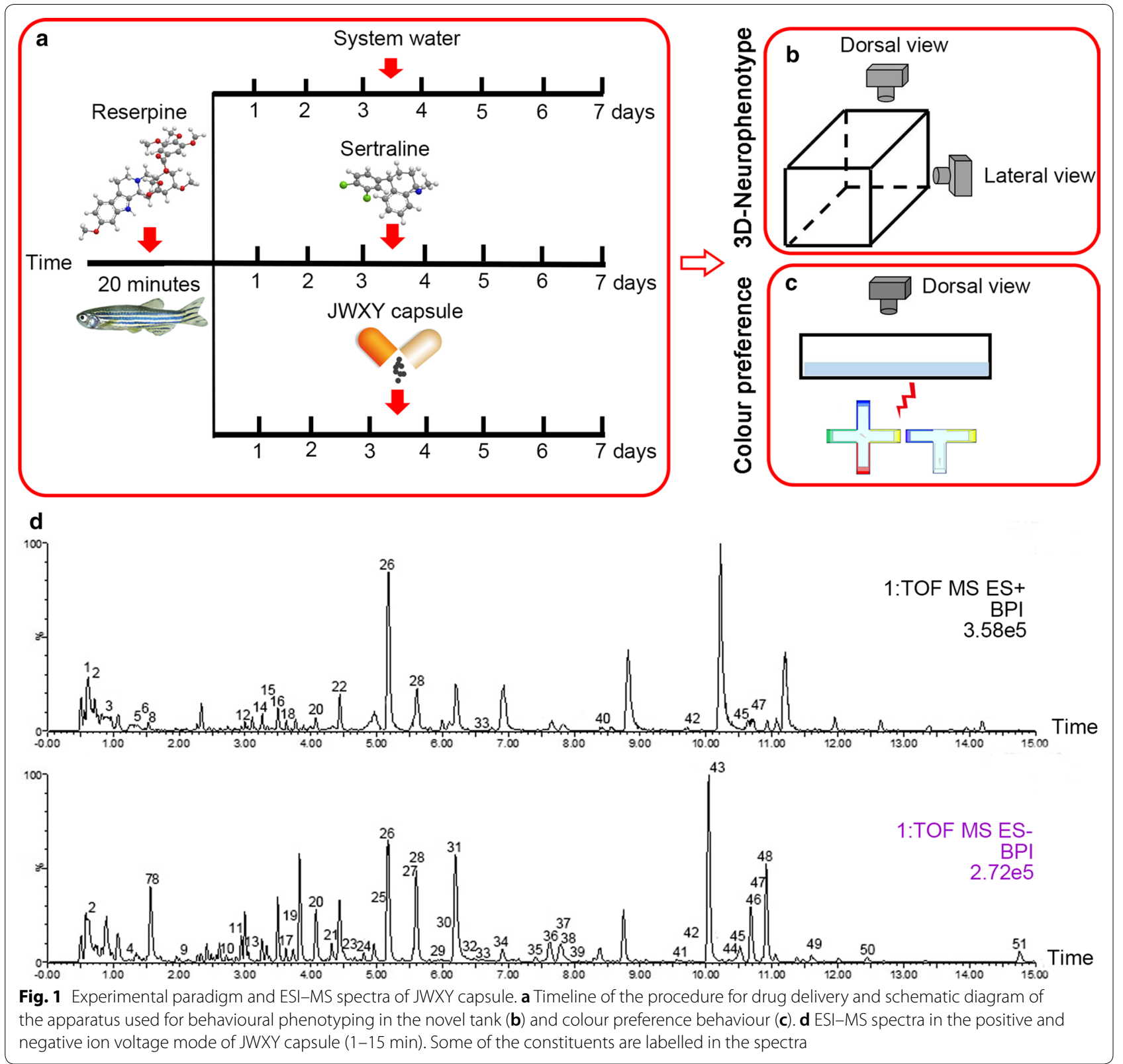

Moutan Cortex, and Gardeniae Fructus (processed with ginger juice). Based on preliminary experiments, the effective concentration of JWXY capsule was $100 \mu \mathrm{g} / \mathrm{mL}$. We opened the capsule and grinded the dry powdered contents. Then, the powdered medicine was weighed and dissolved in system water to obtain a solution with a concentration of $100 \mu \mathrm{g} / \mathrm{mL}$. Experimental solutions were sonicated for $30 \mathrm{~min}$ to dissolve the medication.

A total of 48 experimentally naïve, adult zebrafish (9 months old, male:female $=1: 1$ ) were used in our study. All zebrafish were housed in groups of 2 zebrafish per $4 \mathrm{~L}$ tank (filled with system water maintained at
$28{ }^{\circ} \mathrm{C}$ ) on a $14: 10 \mathrm{~h}$ light cycle. The 48 zebrafish were first tested by 3D neurophenotyping in the novel tank and colour preference behaviour in the maze (defined as control). Then, all 48 zebrafish were exposed $40 \mu \mathrm{g} / \mathrm{mL}$ reserpine for $20 \mathrm{~min}$ and tested by behavioural apparatuses (defined as acute). Next, acute zebrafish were separated into three groups according to the experimental design. The three groups were exposed to system water (indicated as the model), $0.1 \mu \mathrm{g} / \mathrm{mL}$ sertraline hydrochloride (indicated as sertraline) and $100 \mu \mathrm{g} / \mathrm{mL}$ JWXY capsule (indicated as JWXY) for 7 days and then subjected to behavioural testing (Fig. 1a). Solutions were 
Table 1 The definitions of behavioural parameters in the novel tank

\begin{tabular}{|c|c|}
\hline Behavioural parameters & Definition \\
\hline Total distance travelled (m) & The total distance in the novel tank \\
\hline Average velocity $(\mathrm{cm} / \mathrm{s})$ & The direction and magnitude of zebrafish speed in the novel tank \\
\hline Turn angle $\left(^{\circ}\right)$ & The total turning angle of zebrafish in the novel tank \\
\hline Angular velocity $(\% / s)$ & The direction and magnitude of zebrafish angular speed in the novel tank \\
\hline Meandering $(\% / s)$ & The degree of turning vs. travel distance \\
\hline Average entry duration in the top (s) & The amount of time spent at the top of the novel tank during each crossing \\
\hline Distance travelled in the top (m) & The total distance moved in the defined top part in the novel tank \\
\hline Time spent in the top (s) & The total time spent in the top part of the novel tank \\
\hline Latency to enter the top (s) & The amount of time to first cross from the bottom part to the top of the novel tank \\
\hline Number of entries to the top & The number of crosses from the bottom part to the top of the novel tank \\
\hline Time spent ratio of top: bottom & The ratio of the time spent on top over bottom \\
\hline Distance travelled ratio of top: bottom & The ratio of the total distance moved in the top part vs. the bottom \\
\hline Entries ratio of top: bottom & The number of crosses from the bottom part to the top of the novel tank \\
\hline Freezing bouts (frequency) & The total number of instances of immobility (> $1 \mathrm{~s}$ ) during the 5 min test in the novel tank \\
\hline Freezing duration (s) & The duration of all freezing bouts in the novel tank \\
\hline
\end{tabular}

Table 2 The definitions of behavioural parameters in the maze

\begin{tabular}{ll}
\hline Behavioural parameters & Definition \\
\hline Time (\%) & $\begin{array}{l}\text { The ratio of the time zebrafish spent in each arm (colour) to the total time spent in the maze } \\
\text { Distance (\%) }\end{array}$ \\
& \begin{tabular}{l} 
elled in the maze \\
\hline
\end{tabular}
\end{tabular}

refreshed every day after feeding with fresh brine shrimp.

\section{Behavioural testing}

All zebrafish used in our study were acclimated to the laboratory environment. Before the behavioural test at every endpoint, zebrafish were given $1 \mathrm{~h}$ to acclimate to the tank environment. Behavioural testing was performed between 9:00 am and 16:00 pm, i.e., the middle of the light phase of the light cycle, with tanks filled with system water at a temperature ranging from 26 to $28{ }^{\circ} \mathrm{C}$. Zebrafish behaviours were recorded for $5 \mathrm{~min}$ by CCD cameras and evaluated by analysing the behavioural endpoints in Tables 1 and 2.

\section{Enzyme-linked immunosorbent assay (ELISA)}

Cortisol was extracted from zebrafish whole-body homogenates. Adult zebrafish in different treatment groups were weighed and stored at $-80{ }^{\circ} \mathrm{C}$. The whole zebrafish was dissected into small pieces on ice and homogenised in $500 \mu \mathrm{L}$ ELISA Buffer, followed by sonication on ice for $30 \mathrm{~s}$. Diethyl ether was added to samples, which were shaken for $10 \mathrm{~min}$ and centrifuged at $2000 \mathrm{rpm}$ for $15 \mathrm{~min}$ at $4{ }^{\circ} \mathrm{C}$. After storing the samples at $-80{ }^{\circ} \mathrm{C}$ for $15 \mathrm{~min}$, the supernatant was transferred into new tubes. After the diethyl ether evaporated, the extracts were dissolved in $500 \mu \mathrm{L}$ ELISA Buffer and analysed by using the Cortisol ELISA Kit (Cayman, 500360).

The NA, 5-HT and DA concentrations of adult zebrafish brains were analysed by a NA ELISA Kit (CUSABIO, Wuhan, China), 5-HT ELISA Kit (CUSABIO, Wuhan, China), and DA ELISA Kit (CUSABIO, Wuhan, China), respectively. Zebrafish brain tissue was rinsed with $1 \times \mathrm{PBS}$, homogenised in $1 \mathrm{~mL} 1 \times \mathrm{PBS}$ and stored overnight at $-20{ }^{\circ} \mathrm{C}$. After two freeze-thaw cycles, homogenates were centrifuged at $5000 \mathrm{~g}$ for $5 \mathrm{~min}$ at $4{ }^{\circ} \mathrm{C}$. The supernatant was transferred into new tubes and assayed immediately according to the manufacturer's instruction.

\section{Western blot}

Total protein was extracted from adult zebrafish brain tissue with radioimmunoprecipitation assay (RIPA) (CWBIO, Beijing, China) buffer containing phenylmethylsulfonyl fluoride (PMSF) (Sigma-Aldrich). Protein concentrations were quantified using a BCA Protein Assay Kit (CWBIO). Proteins were separated in 10\% sodium dodecyl sulphate-polyacrylamide gel electrophoresis (SDS-PAGE) and transferred to a polyvinylidene fluoride (PVDF) membrane that was blocked 
with Tris-buffered saline (TBS) containing 5\% skim milk for $1 \mathrm{~h}$ at room temperature. Membranes were incubated with mouse anti-TH (1:1000; Millipore) and mouse anti- glyceraldehyde 3-phosphate dehydrogenase (GAPDH) (1:5000; Proteintech) overnight primary antibodies at $4{ }^{\circ} \mathrm{C}$. After being washed with TBS containing $0.05 \%$ Tween-20 (TBST), the membrane was incubated with anti-mouse HRP-conjugated secondary antibody (1:3000; CWBIO). The membrane was then washed with TBS containing 0.05\% Tween-20, and Super Signal West Pico chemiluminescent substrate (Thermo Scientific) was used for detection.

\section{UPLC and Q-TOF-MS analyses}

We opened the JWXY capsule and ground the dry powdered contents. The powdered medicine was dissolved in system water to obtain a solution. Next, we used the mixed solution for ultra-performance liquid chromatography (UPLC) and quadrupole time-of-flight mass spectrometry (Q-TOF-MS) analyses.

A Waters Acquity UPLC System (Waters, MA, USA) equipped with a photodiode array detector was used. The system was controlled by Masslynx V4.1 software (Waters Co.). An Acquity BEHC18 column $(2.1 \times 100 \mathrm{~mm}, 1.7 \mu \mathrm{m}$; Waters Co. $)$ was used for separations. Using Rongchang capsule as an example, a gradient elution of $0.1 \%$ formic acid in water (A) and $0.1 \%$ formic acid in acetonitrile (B) was performed as follows: $2 \% \mathrm{~B}$ was obtained from 0 to $1 \mathrm{~min}, 2-10 \%$ B from 1 to $3 \mathrm{~min}, 10-15 \%$ B from 3 to $7 \mathrm{~min}, 15-30 \%$ B from 7 to $15 \mathrm{~min}, 30-50 \%$ B from 15 to $20 \mathrm{~min}, 50-80 \%$ B from 20 to $23 \mathrm{~min}$, and $80-100 \% \mathrm{~B}$ from 23 to $24 \mathrm{~min}$; In adition, $100 \%$ B was maintained from 24 to $25 \mathrm{~min} ; 100-2 \%$ B was obtained from 25 to $27 \mathrm{~min}$; and $2 \%$ B was maintained from 27 to $30 \mathrm{~min}$. Other samples were slightly adjusted based on their ingredients and chemical polarity. The flow rate was $0.40 \mathrm{~mL} / \mathrm{min}$, and the column temperature was maintained at $35{ }^{\circ} \mathrm{C}$. Accurate mass measurements and MS/MS were performed on a Waters Q-TOF Premier with an electrospray ionisation (ESI) system (Xevo G2-Q Tof, Waters MS Technologies, Manchester, UK). The electrospray ionisation mass spectrometry (ESI-MS) spectra were acquired in both the negative and positive ion voltage modes. The capillary voltages were set to $2.0 \mathrm{kV}$ for the negative mode and $3.0 \mathrm{kV}$ for the positive mode. The sample cone voltage was set to $40 \mathrm{~V}$. Highpurity nitrogen was used as the nebulisation and auxiliary gas. The nebulisation gas was set at a flow rate of $800 \mathrm{~L} / \mathrm{h}$ at $450{ }^{\circ} \mathrm{C}$, the cone gas was set at a flow rate of $50 \mathrm{~L} / \mathrm{h}$, and the source temperature was $120^{\circ} \mathrm{C}$. The Q-TOF Premieracquisition rate was $0.1 \mathrm{~s}$, with a 0.2 -s scan delay. The instrument was operated with the first resolving quadrupole in a wide pass mode (50-2000 Da) and with the collision cell operating at two alternative energies (i.e., 20 and $50 \mathrm{eV})$. Leucine enkephalin $(200 \mathrm{pg} / \mathrm{mL})$ was used as the lock mass $\left([\mathrm{M}-\mathrm{H}]^{-}\right.$554.2615, $\left.[\mathrm{M}+\mathrm{H}]^{+} 556.2771\right)$.

\section{Data analyses}

Data represents the mean \pm SEM (standard error of the mean). One-way ANOVA was performed to assess differences between groups, followed by post hoc Tukey HSD tests for data with a normal distribution. A nonparametric Kruskal-Wallis test followed by Dunn's multiple comparisons tests $(" p<0.05)$ was used for data that violated the assumption of normality. We used GraphPad Prism 7.0 to obtain statistical charts and graphs.

\section{Results}

Establishment of the experimental procedure and analyses of JWXY capsule constituents

All herbs in JWXY capsule are presented in Additional file 1: Table S1. We utilized a solution of capsule contents to conduct a novel tank assay and colour preference behavioural experiment. UPLC and Q-TOF-MS analyses were conducted for JWXY capsule analysis. Protonated $[\mathrm{M}+\mathrm{H}]^{+}$or deprotonated $[\mathrm{M}-\mathrm{H}]^{-}$ions were obtained with as much characteristic fragment information as possible to deduce the molecular and elemental compositions of every constituent. The inferred chemical structure was compared with published data and reported natural product information. The ESI-MS spectra were acquired in both the positive and negative ion voltage modes for each capsule. Here, we show the results of JWXY capsule in the positive and negative ion voltage mode (Fig. 1d). A total of 57 compounds were identified in JWXY capsule. Detailed identification results are presented in Additional file 1: Table S2, Figures S1-S8.

\section{Both sertraline and JWXY capsule rescued the decreased locomotive behaviour of zebrafish caused by reserpine}

The locomotive behaviour of zebrafish was measured by the total distance travelled, average velocity, turn angle and angular velocity. Compared with the control, acute treatment $(20 \mathrm{~min})$ with reserpine resulted in suppression of the total distance travelled and average velocity. Then, after treatment with system water for 7 days, the total distance travelled and average velocity were more significantly reduced (model). The turn angle and angular velocity showed the same trend. However, total distance travelled was rescued after treatment with sertraline and JWXY capsule for 7 days (Fig. 2b). Moreover, the average velocity revealed that sertraline and JWXY capsule significantly rescued the reduced activity caused by reserpine (Fig. 2c). Likewise, the turn angle and angular velocity demonstrated that sertraline and JWXY capsule rescued the effects of reserpine on zebrafish behaviour (Fig. 2d, 

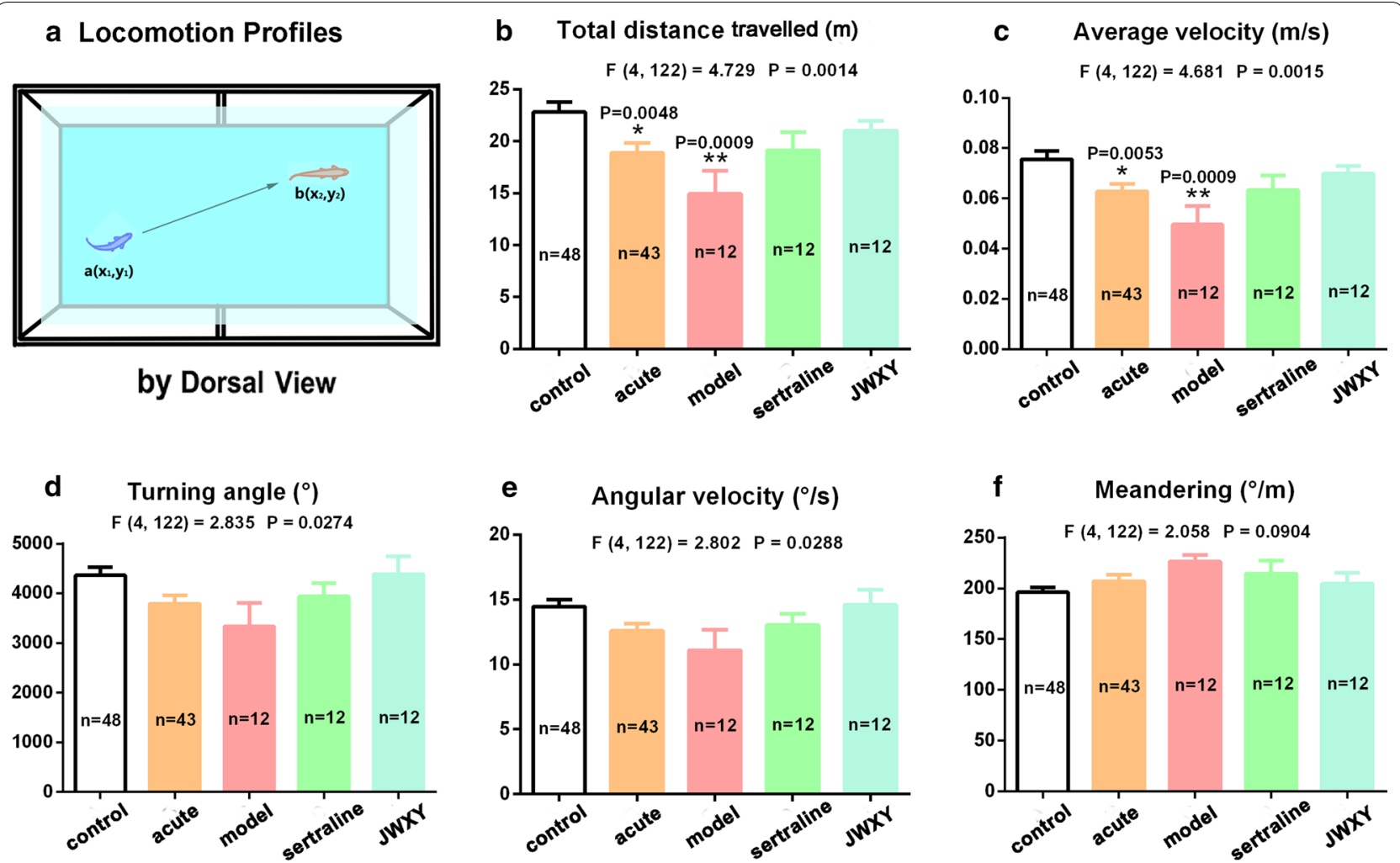

Fig. 2 Locomotion profiles of zebrafish exposed to sertraline and JWXY capsule after reserpine treatment in the novel tank test. a Diagram of locomotion behaviour recorded by a camera from a dorsal view. Histogram revealing the locomotion behaviour of adult zebrafish by the $\mathbf{b}$ total distance travelled, $\mathbf{c}$ average velocity, $\mathbf{d}$ turn angle, e angular velocity and $\mathbf{f}$ meandering. Control: untreated AB strain zebrafish. Acute: acute treatment with reserpine for 20 min. Model: after acute treatment with reserpine, zebrafish were exposed to system water for 7 days to generate the depression model. Sertraline: after acute treatment with reserpine, zebrafish were exposed to sertraline for 7 days. JWXY: after acute treatment with reserpine, zebrafish were exposed to JWXY capsule for 7 days. The data are expressed as the mean \pm S.E.M. and were analysed by one-way ANOVA followed by the Tukey post hoc test. Significance was defined as ${ }^{*} p<0.05,{ }^{* *} p<0.01$

e). Reserpine slightly increased erratic movements measured by meandering in the novel tank test, and sertraline and JWXY capsule reduced this tendency (Fig. 2f).

\section{JWXY capsule rescued inhibition of exploratory behaviour} and reversed the depressive phenotype of zebrafish

Exploratory behaviour, measured by the average entry duration (Fig. 3b), distance travelled in the top (Fig. 3c), time spent in the top (Fig. $3 \mathrm{~d}$ ), time spent ratio of top: bottom (Fig. 3e), distance travelled of top: bottom (Fig. 3f), latency to enter the top (Fig. 3g) and entries ratio of top: bottom (Fig. 3h), was not significantly altered in the treatment groups compared with that in the control group, with the exception of JWXY capsule treatment group. However, exploratory behavioural parameters were decreased in the model group. As shown in Fig. 3, zebrafish treated with JWXY capsule exhibited improvements in exploratory behaviour; the average entry duration, distance travelled in the top, time spent in the top, time spent ratio of top: bottom and distance travelled of top: bottom were significantly higher in the JWXY capsule group than in the acute group. Reserpine induced an obvious depressive phenotype as shown in Fig. 4. After acute treatment with reserpine, zebrafish did not show changes in their freezing bouts and freezing duration. However, the freezing bouts and freezing duration were enhanced after 7 days. After treatment with of sertraline and JWXY capsule for 7 days, the depressive phenotype was no longer observed. Moreover, the freezing bouts and freezing duration were significantly decreased.

\section{Impact of sertraline and JWXY capsule on the colour preference behaviour of zebrafish after reserpine treatment: JWXY capsule reversed colour preference patterns}

Colour preference behaviour was demonstrated by the time spent (Fig. 5) and distance travelled (Additional file 1: Figure S9) in every colour arm using a remoulded offset cross maze (Fig. 5a). The control group (Fig. 5b) spent the most time in the blue area, followed by the 


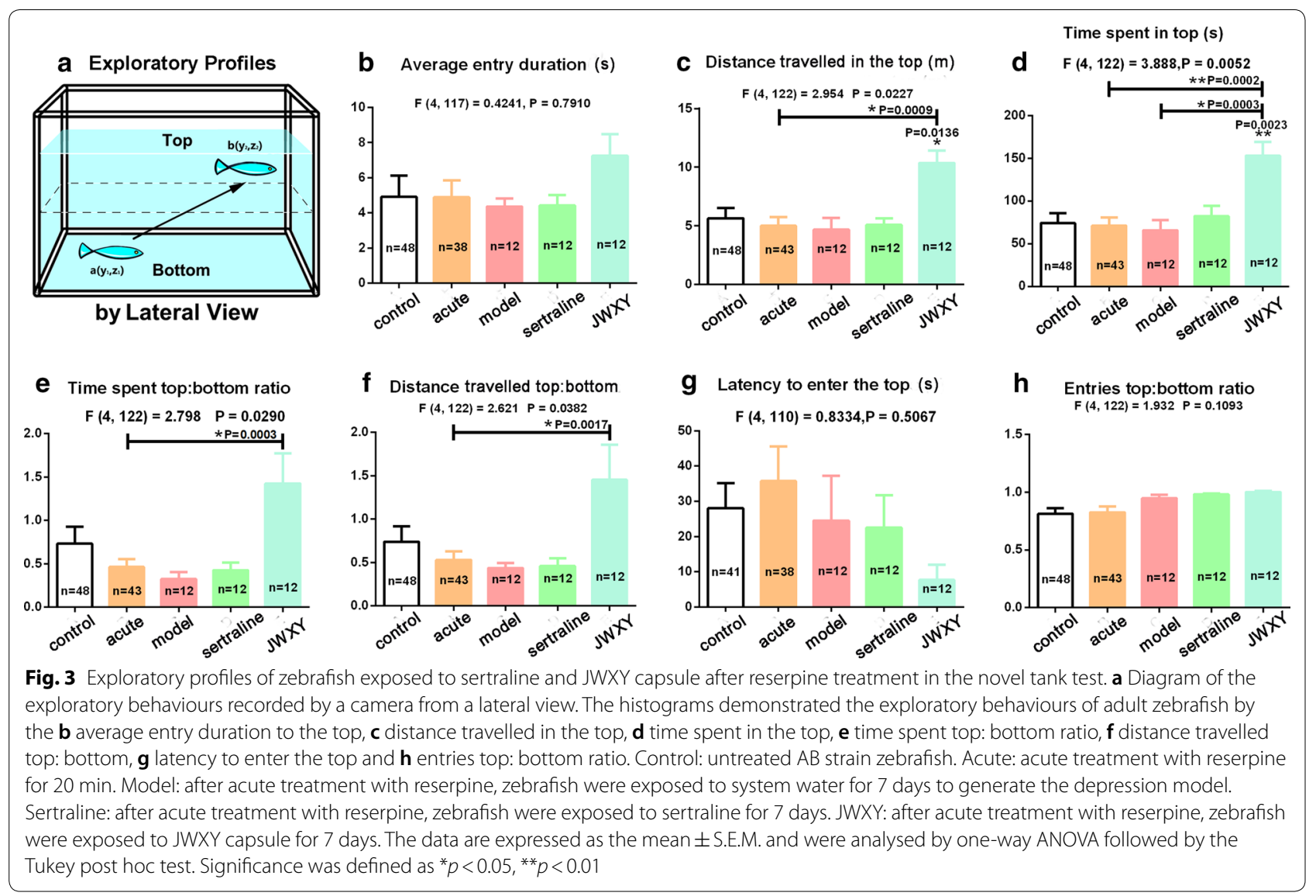

\section{a} Freezing bouts (frequency)

$F(4,122)=4.724 P=0.0014$

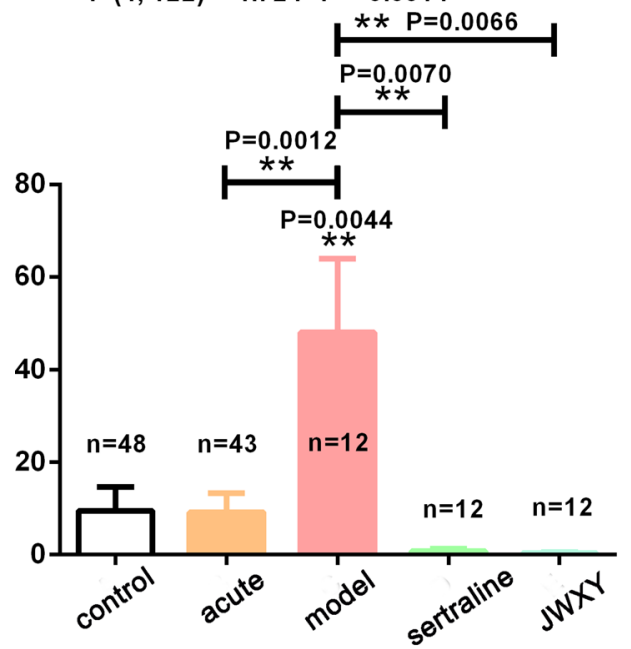

b

Freezing duration (s)

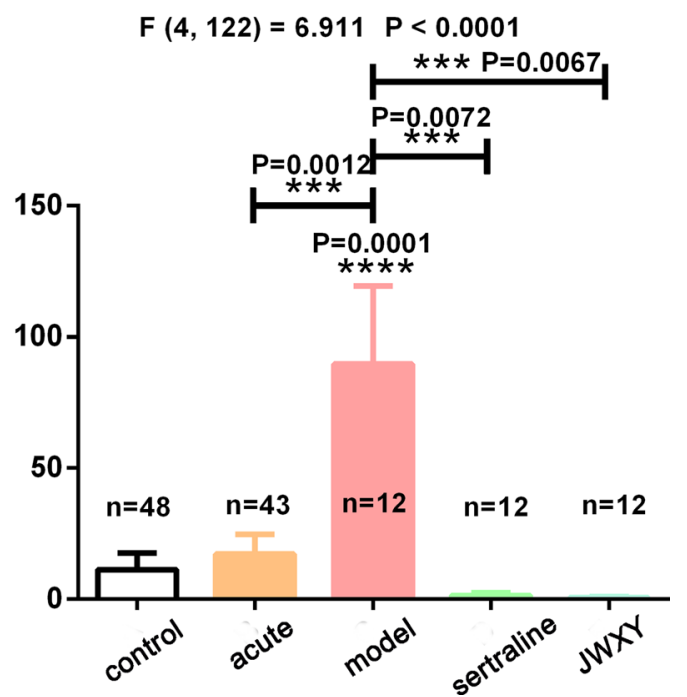

Fig. 4 Histogram of the $\mathbf{a}$ freezing bouts and $\mathbf{b}$ freezing duration of zebrafish. Control: untreated AB strain zebrafish. Acute: acute treatment with reserpine for 20 min. Model: after acute treatment with reserpine, zebrafish were exposed to system water for 7 days to generate the depression model. Sertraline: after acute treatment with reserpine, zebrafish were exposed to sertraline for 7 days. JWXY: after acute treatment with reserpine, zebrafish were exposed to JWXY capsule for 7 days. The data are expressed as the mean \pm S.E.M. and were analysed by one-way ANOVA followed by the Tukey post hoc test. Significance was defined as ${ }^{*} p<0.05,{ }^{* *} p<0.01,{ }^{* * *} p<0.001$ and ${ }^{* * * *} p<0.0001$ 


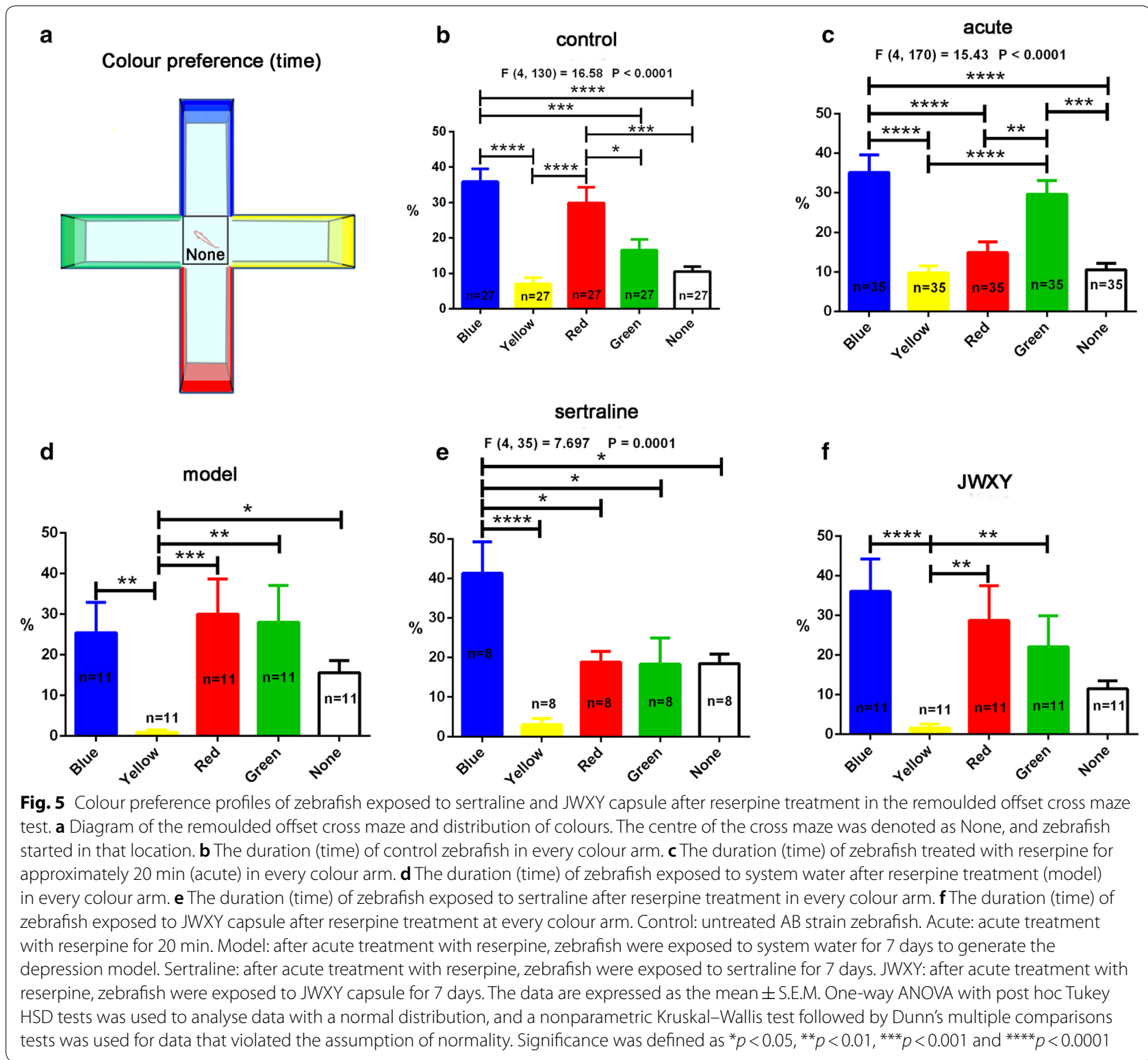

red, green, and yellow areas (listed from most to least time spent). The most and least preferred colours were unchanged, but the time spent in green area was higher than that in the red area in the acute group (Fig. 5c). In the model group (Fig. 5d), the amounts of time spent in blue, red and green were not significantly different. Zebrafish continued to spend the least amount of time in the yellow area. After sertraline treatment (Fig. 5e), zebrafish recovered their preference for blue. However, the time spent in the red and green areas was not different. Notably, the JWXY group and control group had the same colour preference (Fig. 5f). The distance (Additional file 1: Figure S9) travelled in every colour arm was the same over time. Based on the abovementioned results, we concluded that zebrafish preferred blue the most and yellow the least. Following treatment with sertraline and JWXY capsule, the colour preference order was restored, and JWXY capsule was more effective than sertraline in restoring colour preference behaviour.

Based on colour preference behaviour in the remoulded offset cross maze, we chose two colours (blue and yellow) to conduct a concise test using a T-maze. Colour preference behaviour was demonstrated by time (Fig. 6) and distance (Additional file 1: Figure S10) travelled in every colour arm using a T-maze (Fig. 6a). In the control (Fig. 6b) and acute (Fig. 6c) groups, zebrafish spent 


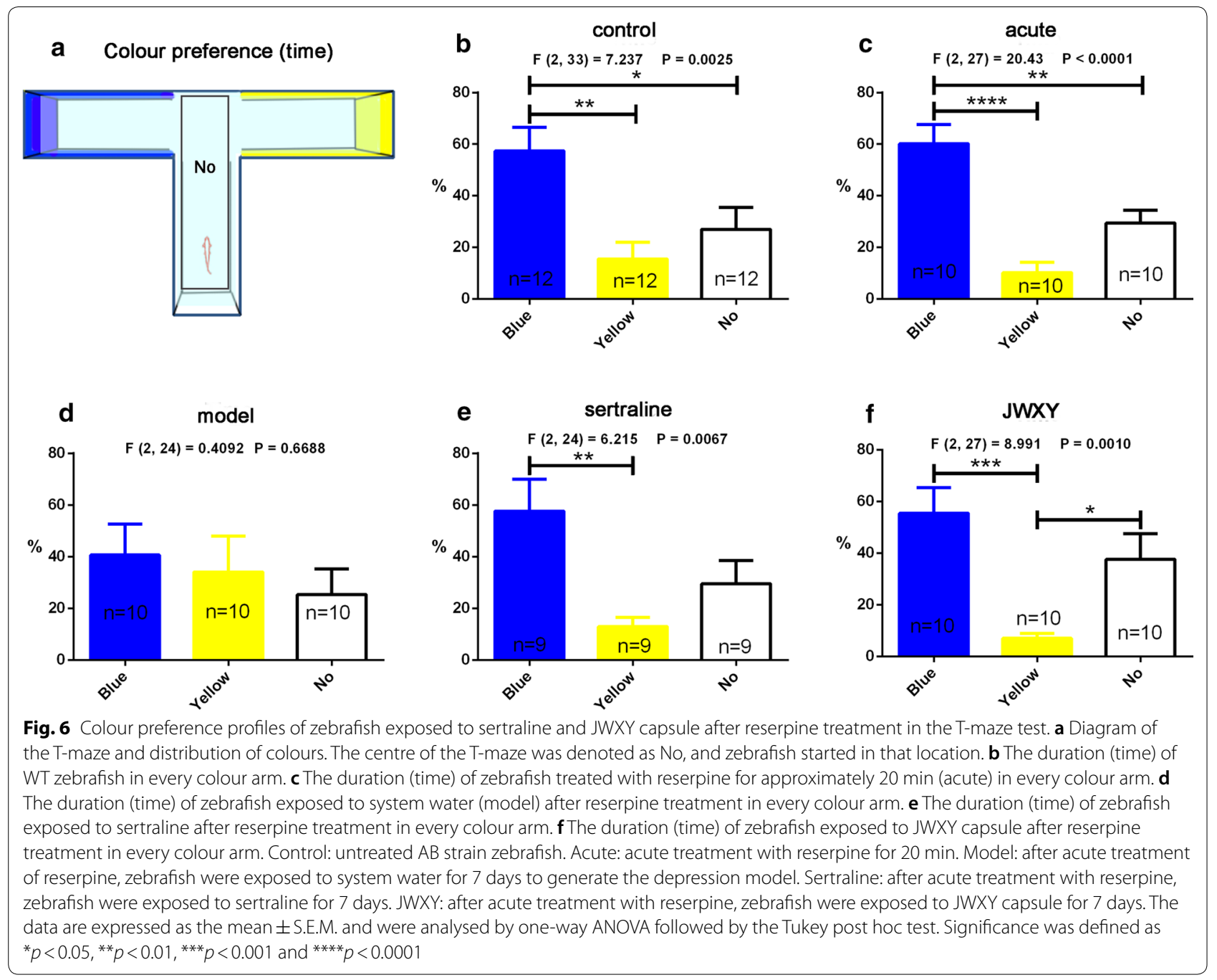

significantly more time in the blue area than in the yellow area. However, the time zebrafish spent in every colour arm was not different in the model group (Fig. 6d). After sertraline (Fig. 6e) and JWXY capsule (Fig. 6f) treatment, the same colour preference tendency as that of the control was observed. The distance (Additional file 1: Figure S10) travelled in every colour arm was consistent with the time spent.

\section{Cortisol and monoamine levels influenced zebrafish neurobehaviour}

Whole-body cortisol, monoamines, including NA, 5-HT, $\mathrm{DA}$, and $\mathrm{TH}$ were detected in zebrafish brain tissues after different treatments. The cortisol level in the model group was significantly higher than that in the acute and control groups. After sertraline and JWXY capsule treatments, the cortisol level was markedly decreased, especially in the JWXY group (Fig. 7a). NA in the model group was significantly lower than that in the control and sertraline group. However, compared with the model and sertraline groups, the JWXY group showed a significant increase in the NA concentration (Fig. 7b). 5-HT was significantly increased in the acute group but increased only slightly in the model group. Sertraline elevated the 5-HT level, but JWXY capsule did not (Fig. 7c). Sertraline decreased TH expression 7 days after acute exposure, and sertraline and JWXY capsule treatments improved TH to a degree (Fig. 7d). However, the DA level did not change after sertraline and JWXY treatments (Additional file 1: Figure S11).

\section{Discussion}

JWXY capsule contains nine herbal medicines, and 57 compounds were identified in its extraction by UPLC and Q-TOF-MS. As previously described, TCM posits that depression involves in multiple organs. TCM focuses on the overall effect of medicines contained in a prescription, and it also plays a role in health care and disease 

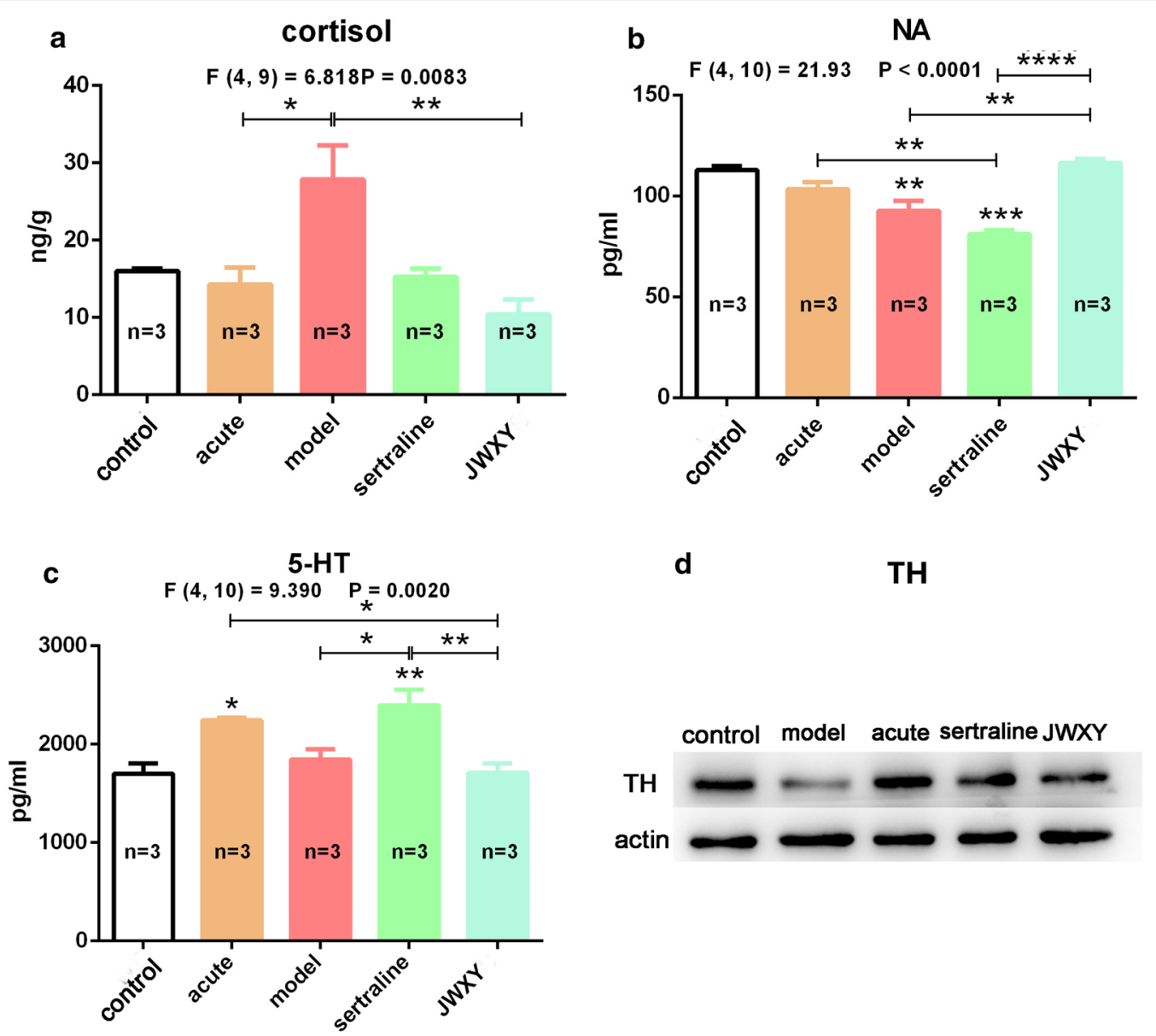

Fig. 7 The effects of sertraline and JWXY capsule treatment on cortisol and monoamines in zebrafish. The levels of cortisol (a), noradrenaline (b), 5-HT (c) and tyrosine hydroxylase (d) in zebrafish after different treatments. Control: untreated AB strain zebrafish. Acute: acute treatment with reserpine for $20 \mathrm{~min}$. Model: after acute treatment with reserpine, zebrafish were exposed to system water for 7 days to generate the depression model. Sertraline: after acute treatment with reserpine, zebrafish were exposed to sertraline for 7 days. JWXY: after acute treatment with reserpine, zebrafish were exposed to JWXY capsule for 7 days. The data are expressed as the mean \pm S.E.M. and were analysed by one-way ANOVA followed by the Tukey post hoc test. Significance was defined as ${ }^{*} p<0.05,{ }^{* *} p<0.01,{ }^{* * *} p<0.001$ and ${ }^{* * *} p<0.0001$

prevention through the treatment of multiple targets. All herbal medicines in the prescription work synergistically and can yield stable and comprehensive curative effects, greatly reducing the side effects of drug treatment. Determining the main components of TCM prescriptions and their mechanisms of action is difficult. However, characterising the multiple constituents, targets and pathways of TCM prescriptions is of greater importance, and requires further research.

We employed a 3D video-tracking system to detect changes in the swimming behaviour of lesioned zebrafish in novel tank. Zebrafish demonstrated longterm depressive symptoms, including elevated baseline whole-body cortisol, social withdrawal and locomotor retardation after reserpine exposure [46]. Reserpine does not induce overt acute behavioural effects but markedly reduces activity after 7 days [5], consistent with our study. After 20 min of reserpine exposure, zebrafish showed a slight decrease in locomotive activity but did not show obvious changes in exploratory behaviour and freezing behaviour. Zebrafish displayed significantly decreased locomotive activity and a worsened depressive phenotype after 7 days, along with hypoactive exploratory behaviour, which proved that the establishment of zebrafish depression model first introduced by Kyzar et al. was successful in our experiment. However, compared with zebrafish in the model group, zebrafish in the sertraline and JWXY capsule groups treated for 7 days expressed different behaviours. Sertraline treatment increased locomotive activity and rescued the depressive phenotype induced by reserpine. Moreover, JWXY capsule increased locomotive activity, more effectively reversed the depressive phenotype, and improved exploratory behaviour. 
The colour preference test could serve as a useful protocol for memory evaluation, cognitive dysfunction, assessment of neurodegenerative disorders, preclinical appraisal of drug efficacy and behavioural evaluation of toxicity [47]. Here, we evaluated cognitive impairment by the colour preference test. Studies have demonstrated the natural colour preference of zebrafish. Zebrafish prefer colours of short wavelengths. Zebrafish exhibit a strong preference for blue relative to all other colours (red, yellow and green), with yellow being less preferred than red and green $[26,48]$. In our study, blue was the favourite colour of control zebrafish, and yellow was the least favourite. Control zebrafish exhibited a significantly stronger preference for blue than for red and green. However, compared with control zebrafish, zebrafish exhibiting depressive behaviour lost certain colour preferences. Yellow was the least preferred colour of model zebrafish, but the preference for green and red increased simultaneously and was not significantly different compared with that for blue, indicating that the normal colour preference pattern was disturbed. However, Zebrafish treated by JWXY capsule regained this colour preference pattern. Sertraline also restored the colour preference pattern to a degree, but its efficacy was not as obvious and clear as that of JWXY capsule. To minimize the effects of place preference on the results and further verify this preference in zebrafish, we chose to test blue and yellow in T-maze. All groups except the model group exhibited a preference for blue. However, different from the other groups, the model group also showed an increased preference for yellow and the same preference for all three arms, illustrating the colour preference disorder in depressed zebrafish. In contrast, sertraline and JWXY capsule restored the colour preference pattern. These results showed that the cognitive dysfunction accompanying with depression in zebrafish could be reversed by sertraline and JWXY capsule.

Depression is usually comorbid with anxiety, which leads to behavioural alterations. The effects of chronic depression and anxiety on the hypothalamic-pituitary-interrenal (HPI) axis have been studied previously in zebrafish. Benzodiazepines (anxiolytics) and antidepressants completely prevent increased cortisol levels in zebrafish [49]. The decreases in total distance travelled and velocity in zebrafish are related to the decreased levels of DA and NA [50]. SSRIs were developed and entered clinical trials as a new class of antidepressant in the 1980s. Six SSRIs, including fluoxetine, paroxetine, sertraline, fluvoxamine, citalopram and escitalopram are commonly used for clinical treatment. SSRIs selectively inhibit the reuptake of 5-HT by the presynaptic membrane. SSRIs have little impact on NA and hardly affect the reuptake of DA [51]. In our study, the reserpine-induced zebrafish model of depression showed increased whole-body cortisol and 5-HT, decreased NA and reduced $\mathrm{TH}$. Compared with the model, sertraline prevented the increase in cortisol and NA and increased 5-HT and TH. However, JWXY capsule prevented the increase in cortisol and 5-HT, consistent with the rescued depressive phenotype. In addition, compared with the model, JWXY capsule improved the levels of NA and $\mathrm{TH}$, consistent with the increased locomotive activity. Interestingly, DA levels in zebrafish brains were unaffected by any treatments. Those changes in monoamine neurotransmitters were related to the colour preference disorder caused by reserpine and were consistent with the restored cognitive ability.

\section{Conclusion}

The novel tank test recorded by a 3D method in this experiment revealed the similar anti-depression effects of two treatments for chronic reserpine exposure. This validation was based on the successful establishment of a depressive zebrafish model, which was first introduced by Kyzar et al. The depressive effects of reserpine decrease locomotion, increase erratic movements, reduce exploratory behaviour to the top and enhance depressive phenotype. Furthermore, colour preference testing in a remoulded offset cross maze and T-maze indicated that the natural colour preference pattern (zebrafish prefer blue to red, green and yellow and show a strong aversion to yellow) was disturbed due to depression induced by reserpine. However, sertraline treatment improved depression-like behaviours by increasing locomotion and decreasing erratic movements and the depressive phenotype. Sertraline also restored the colour preference in zebrafish. Notably, JWXY capsule was a more effective treatment than sertraline. JWXY capsule treatment reversed depression-like behaviours by increasing locomotion, decreasing erratic movements, increasing exploratory behaviour to the top and rescued the depressive phenotype. Zebrafish also exhibited their natural colour preference after JWXY capsule treatment.

Depression-like behaviours and cognitive disorder (measured by colour preference) resulted from changes in hormone and monoamine neurotransmitters in the brain. Increased whole-body cortisol and decreased NA and $\mathrm{TH}$ were observed in the zebrafish depression model. Sertraline prevented the increase in cortisol, inhibited the reuptake of 5-HT, and improved the expression of TH. Compared with the model, JWXY capsule also prevented the increase in cortisol, recovered NA and improved the expression of $\mathrm{TH}$. Overall, these results show that changes in cortisol and monoamines accounted for the reversal of depressive behaviours and cognitive dysfunction. The high sensitivity of zebrafish to the effects of Western medicine and TCM 
can help improve our understanding of the psychopharmacological profiles of these drugs and related CNS drugs, as well contribute to further development of TCM as an antidepressant.

\section{Additional file}

Additional file 1: Table S1. The composition of JWXY capsule. Figure S1. ESI-MS spectra in the positive and negative ion voltage mode of JWXY capsule (1-30 min). Figure S2. ESI-MS spectra in the positive and negative ion voltage mode of JWXY capsule (15-30 min). Table S2. MS data in $( \pm)$ ESI modes and the identification results in JWXY capsule. Figures S3-8. The chemical structure of each component identified in JWXY capsule. Figure S9. Colour preference profiles of zebrafish exposed to sertraline and JWXY capsule after reserpine treatment in the remoulded offset cross maze test. Figure S10. Colour preference profiles of zebrafish exposed to sertraline and JWXY capsule after reserpine treatment in the T-maze test. Figure S11. The changes of sertraline and JWXY capsule treatment on dopamine (DA) of zebrafish.

\section{Authors' contributions}

$X Z F, X D L$ and $X L$ conceived and designed the experiments. XDL, SHZ and XL conducted the zebrafish behavioural assays. SHZ and XDL wrote the manuscript. The video-tracking software was designed by MZS, TL and XZ. XDL and SHZ collected and analysed the behavioural data. QPZ, JX and DYC monitored and evaluated the expression of related hormones and monoamines. All authors discussed the results and implications and reviewed the manuscript at all stages. All authors read and approved the final manuscript.

\section{Acknowledgements}

This work was supported by the Special Fund for Basic Research on Scientific Instruments from the Chinese National Natural Science Foundation of China (Grant No: 61327802), the National Basic Research Program of China (2015CB856500) and the Chinese National Natural Science Foundation of China (Grant Nos. 61633012 and U1613220).

\section{Competing interests}

The authors declare that they have no competing interests.

\section{Consent for publication}

All authors have read and approved this version of the article for publication.

\section{Availability of data and materials}

The datasets used and/or analyzed in this study are available from the corresponding author on reasonable request.

\section{Ethics approval and consent to participate}

All of the experimental protocols and procedures involving zebrafish were approved by the Committee for Animal Experimentation of the College of Life Science at Nankai University (No. 2008) and were performed in accordance with the NIH Guide for the Care and Use of Laboratory Animals (No. 8023, revised in 1996).

\section{Publisher's Note}

Springer Nature remains neutral with regard to jurisdictional claims in published maps and institutional affiliations.

Received: 1 January 2018 Accepted: 1 June 2018

Published online: 14 June 2018

\section{References}

1. Simon NM. Generalized anxiety disorder and psychiatric comorbidities such as depression, bipolar disorder, and substance abuse. J Clin Psychiatry. 2009;70(suppl 2):10-4.
2. Richelson E. Pharmacology of antidepressants. Mayo Clin Proc. 2001;76(5):511-27.

3. Perrine SA, Ghoddoussi F, Michaels MS, Sheikh IS, Mckelvey G, Galloway MP. Ketamine reverses stress-induced depression-like behaviour and increased GABA levels in the anterior cingulate: an 11.7 T 1H-MRS study in rats. Prog Neuropsychopharmacol Biol Psychiatry. 2014;51(1):9-15.

4. McCarroll MN, Gendelev L, Keiser MJ, Kokel D. Leveraging large-scale behavioural profiling in zebrafish to explore neuroactive polypharmacology. ACS Chem Biol. 2016;11(4):842-9.

5. Kyzar E, Stewart AM, Landsman S, Collins C, Gebhardt M, Robinson K, Kalueff AV. Behavioural effects of bidirectional modulators of brain monoamines reserpine and d-amphetamine in zebrafish. Brain Res. 2013;1527:108-16.

6. Goldstein DS, Eisenhofer G, Mccarty R. Catecholamines: bridging basic science with clinical medicine. Cambridge: Academic Press; 1998.

7. Lillesaar C. The serotonergic system in fish. J Chem Neuroanat. 2011:41(4):294-308.

8. Yamamoto K, Vernier P. The evolution of dopamine systems in chordates. Front Neuroanat. 2011;5(5):1-21.

9. Vignet C, Trenkel VM, Vouillarmet A, Bricca G, Begout ML, Cousin X. Changes in brain monoamines underlie behavioural disruptions after zebrafish diet exposure to polycyclic aromatic hydrocarbons environmental mixtures. Int J Mol Sci. 2017;18(3):560.

10. Levin ED, Kalueff AV, Gerlai RT. Perspectives on zebrafish neurobehavioural pharmacology. Pharmacol Biochem Behav. 2015;139:93.

11. Kalueff AV, Stewart AM, Gerlai R. Zebrafish as an emerging model for studying complex brain disorders. Trends Pharmacol Sci. 2014;35(2):63.

12. Saroya R, Smith $R$, Seymour C, Mothersill C. Injection of resperpine into zebrafish, prevents fish to fish communication of radiation-induced bystander signals: confirmation in vivo of a role for serotonin in the mechanism. Doseresponse Publ Int Hormesis Soc. 2009:8(3):317-30.

13. Fossat P, Bacqué-Cazenave J, De DP, Delbecque JP, Cattaert D. Comparative behaviour. Anxiety-like behaviour in crayfish is controlled by serotonin. Science. 2014;344(6189):1293-7.

14. Maximino C, Puty B, Benzecry R, Araujo J, Lima MG, de Jesus Oliveira Batista E, de Matos Oliveira KR, Crespo-Lopez ME, Herculano AM. Role of serotonin in zebrafish (Danio rerio) anxiety: relationship with serotonin levels and effect of buspirone, WAY 100635, SB 224289, fluoxetine and para-chlorophenylalanine (pCPA) in two behavioural models. Neuropharmacology. 2013;71:83-97.

15. Abril-de-Abreu R, Cruz J, Oliveira RF. Social Eavesdropping in Zebrafish: tuning of Attention to Social Interactions. Scientific Rep. 2015;5:12678.

16. Li X, Liu B, Li XL, Li YX, Sun MZ, Chen DY, Zhao X, Feng XZ. SiO2 nanoparticles change colour preference and cause Parkinson's-like behaviour in zebrafish. Scientific Rep. 2014;4:3810.

17. Khotimah H, Sumitro SB, Widodo MA. Zebrafish Parkinson's model: rotenone decrease motility, dopamine, and increase a-synuclein aggregation and apoptosis of zebrafish brain. Int J Pharmtech Res. 2015;8(4):614-21.

18. Wang YN, Hou YY, Sun MZ, Zhang CY, Bai G, Zhao X, Feng XZ. Behavioural screening of zebrafish using neuroactive traditional Chinese medicine prescriptions and biological targets. Scientific Rep. 2014;4:5311.

19. Anderson HD, Pace WD, Libby AM, West DR, Valuck RJ. Rates of 5 common antidepressant side effects among new adult and adolescent cases of depression: a retrospective US claims study. Clin Ther. 2012;34(1):113.

20. Rascati K, Godley P, Pham H. Evaluation of resources used to treat adverse events of selective serotonin reuptake inhibitor use. J Manag Care Pharm. 2001;7:402-6.

21. Dai Y, Li Z, Xue L, Dou C, Zhou Y, Zhang L, Qin X. Metabolomics study on the anti-depression effect of xiaoyaosan on rat model of chronic unpredictable mild stress. J Ethnopharmacol. 2010;128(2):482-9.

22. Zhou J. Multicenter randomized controlled clinical study of JiaWeiXiaoYao capsule in the treatment of mild to moderate depression with syndrome of qi stagnation transforming into fire. China Academy of Chinese Medical Sciences; 2013.

23. Zhou J, Rui SU, Tao LI, Cao XD, Han ZY, Lin B, Guo RJ, Fan JP. Randomized controlled trial of Jiawei Xiaoyao Capsule in the treatment of mild to moderate depression. China J Trad Chin Med Pharm. 2013;28(9):2804-6.

24. Park JS, Ryu JH, Choi TI, Bae YK, Lee S, Kang HJ, Kim CH. Innate colour preference of zebrafish and its use in behavioural analyses. Mol Cells. 2016;39(10):750-5. 
25. Braida D, Ponzoni L, Martucci R, Sparatore F, Gotti C, Sala M. Role of neuronal nicotinic acetylcholine receptors ( $\mathrm{nAChRs)}$ on learning and memory in zebrafish. Psychopharmacology. 2014;231(9):1975-85.

26. Avdesh A, Martin-Iverson M, Chen M, Groth D, Mondal A, Morgan $\mathrm{N}$, Lardelli M, Martins R, Verdile G. Evaluation of colour preference in zebrafish: a possible potential model for learning and memory disorders. Alzheimers Dementia J Alzheimers Assoc. 2011;7(4):S120-S120.

27. Li X, Li X, Li YX, Zhang Y, Chen D, Sun MZ, Zhao X, Chen DY, Feng XZ. The Difference between Anxiolytic and Anxiogenic Effects Induced by Acute and Chronic Alcohol Exposure and Changes in Associative Learning and Memory Based on Colour Preference and the Cause of Parkinson-Like Behaviours in Zebrafish. PLoS ONE. 2015;10(11):e0141134.

28. Oliveira J, Silveira M, Chacon D, Luchiari A. The zebrafish world of colours and shapes: preference and discrimination. Zebrafish. 2015;12(2):166-73.

29. Robinson J, Schmitt EA, Hárosi Fl, Reece RJ, Dowling JE. Zebrafish ultraviolet visual pigment: absorption spectrum, sequence, and localization. Proc Natl Acad Sci USA. 1993;90(13):6009-12.

30. Fadool JM, Dowling JE. Zebrafish: a model system for the study of eye genetics. Prog Retinal Eye Res. 2008;27(1):89-110.

31. Roest $\mathrm{CH}$, Weissenbach J. Fish genomics and biology. Genome Res. 2005; 15(12):1675-82.

32. Stewart AM, Ullmann JF, Norton WH, Parker MO, Brennan CH, Gerlai R, Kalueff AV. Molecular psychiatry of zebrafish. Mol Psychiatry. 2015:20(1):2-17.

33. Cheng KC, Xin X, Clark DP, Riviere PL. Whole-animal imaging, gene function, and the Zebrafish Phenome Project. Curr Opin Genet Dev. 2011:21(5):620-9.

34. Rihel J, Prober DA, Arvanites A, Lam K, Zimmerman S, Jang S, Haggarty SJ, Kokel D, Rubin LL, Peterson RT, Schier AF. Zebrafish behavioural profiling links drugs to biological targets and rest/wake regulation. Science. 2010:327(5963):348-51.

35. Bruni G, Rennekamp AJ, Velenich A, McCarroll M, Gendelev L, Fertsch E, Taylor J, Lakhani P, Lensen D, Evron T, Lorello PJ, Huang XP, Kolczewski S, Carey G, Caldarone BJ, Prinssen E, Roth BL, Keiser MJ, Peterson RT, Kokel D. Zebrafish behavioural profiling identifies multitarget antipsychotic-like compounds. Nat Chem Biol. 2016;12(7):559-66.

36. Challal S, Buenafe OEM, Queiroz EF, Maljevic S, Marcourt L, Bock M, Kloeti W, Dayrit FM, Harvey AL, Lerche H, Esguerra CV, de Witte PAM, Wolfender $J$-L, Crawford AD. Zebrafish bioassay-guided microfractionation identifies anticonvulsant steroid glycosides from the philippine medicinal plantsolanum torvum. ACS Chem Neurosci. 2014;5(10):993-1004.

37. Sourbron J, Smolders I, de Witte P, Lagae L. Pharmacological analysis of the anti-epileptic mechanisms of fenfluramine in scn1a mutant zebrafish. Front Pharmacol. 2017:8:191.

38. Rihel J, Schier AF. Behavioural screening for neuroactive drugs in zebrafish. Dev Neurobiol. 2012;72(3):373-85.
39. Li X, Li X, Chen D, Guo J-L, Feng D-F, Sun M-Z, Lu Y, Chen D-Y, Zhao X, Feng $X-Z$. Evaluating the biological impact of polyhydroxyalkanoates (PHAs) on developmental and exploratory profile of zebrafish larvae. RSC Adv. 2016;6(43):37018-30.

40. Heilmann S, Ratnakumar K, Langdon EM, Kansler ER, Kim IS, Campbell NR, Perry EB, McMahon AJ, Kaufman CK, van Rooijen E, Lee W, lacobuzioDonahue CA, Hynes RO, Zon LI, Xavier JB, White RM. A quantitative system for studying metastasis using transparent zebrafish. Can Res. 2015;75(20):4272-82.

41. Sokolova MV, Fernández-Caballero A, Ros L, Latorre JM, Serrano JP. Evaluation of color preference for emotion regulation. Artificial computation in biology and medicine, vol. 9107. Heidelberg: Springer; 2015. p. 479-87. https://doi.org/10.1007/978-3-319-18914-7_50

42. Pittman JT, Lott CS. Startle response memory and hippocampal changes in adult zebrafish pharmacologically-induced to exhibit anxiety/depression-like behaviours. Physiol Behav. 2014;123:174-9.

43. Macri S, Neri D, Ruberto T, Mwaffo V, Butail S, Porfiri M. Three-dimensional scoring of zebrafish behaviour unveils biological phenomena hidden by two-dimensional analyses. Scientific Rep. 2017;7(1):1962.

44. Kalueff AV, Cachat JM. Zebrafish neurobehavioural protocols. New York: Humana Press; 2011.

45. Li X, Liu X, Li T, Li X, Feng D, Kuang X, Xu J, Zhao X, Sun M, Chen D, Zhang Z, Feng X. SiO2nanoparticles cause depression and anxiety-like behaviour in adult zebrafish. RSC Adv. 2017;7(5):2953-63.

46. Nguyen M, Stewart AM, Kalueff AV. Aquatic blues: modeling depression and antidepressant action in zebrafish. Prog Neuropsychopharmacol Biol Psychiatry. 2014;55:26-39.

47. Jia L, Raghupathy RK, Albalawi A, Zhao Z, Reilly J, Xiao Q, Shu X. A colour preference technique to evaluate acrylamide-induced toxicity in zebrafish. Comp Biochem Physiol C Toxicol Pharmacol. 2017;199:11-9.

48. Bault ZA, Peterson SM, Freeman JL. Directional and colour preference in adult zebrafish: implications in behavioural and learning assays in neurotoxicology studies. J Appl Toxicol JAT. 2015:35(12):1502-10.

49. Marcon M, Herrmann AP, Mocelin R, Rambo CL, Koakoski G, Abreu MS, Conterato GM, Kist LW, Bogo MR, Zanatta L, Barcellos LJ, Piato AL. Prevention of unpredictable chronic stress-related phenomena in zebrafish exposed to bromazepam, fluoxetine and nortriptyline. Psychopharmacology. 2016;233(21-22):3815-24.

50. Anichtchik OV, Kaslin J, Peitsaro N, Scheinin M, Panula P. Neurochemical and behavioural changes in zebrafish Danio rerio after systemic administration of 6-hydroxydopamine and 1-methyl-4-phenyl-1,2,3,6tetrahydropyridine. J Neurochem. 2004:88(2):443-53.

51. Baldwin D, Buis C, Mayers A. Selective serotonin reuptake inhibitors in the treatment of generalized anxiety disorder. Expert Rev Neurother. 2002;2(5):717-24.

\footnotetext{
Ready to submit your research? Choose BMC and benefit from:

- fast, convenient online submission

- thorough peer review by experienced researchers in your field

- rapid publication on acceptance

- support for research data, including large and complex data types

- gold Open Access which fosters wider collaboration and increased citations

- maximum visibility for your research: over 100M website views per year
}

At BMC, research is always in progress.

Learn more biomedcentral.com/submissions 